\title{
colaboraciones
}

\section{UN SISTEMA DE SANEAMIENTO PARA MADRID}

\section{(SANITATION SYSTEM FOR MADRID)}

\section{Félix Cristóbal Sánchez}

Ingeniero de Caminos, Canales y Puertos

533-27

\section{RESUMEN}

El Plan de Saneamiento Integral de Madrid representaba la culminación de una serie de acciones emprendidas, durante varios años, para conseguir que la ciudad completase su sistema de saneamiento. Con este conjunto de acciones comprendidas en el Plan puede decirse que esta

infraestructura básica ha adquirido un nivel suficiente, capaz de prestar un servicio acorde con las auténticas necesidades de la Villa.

\section{SUMMARY}

The Full Sanitation Plan for Madrid is the final action of a serie undertaken for several years with the purpose to reach the completion of the sanitation system of the town. With the whole of these actions it could be said that the basic substructure has acquired enough level to deserve de real needs of the town.

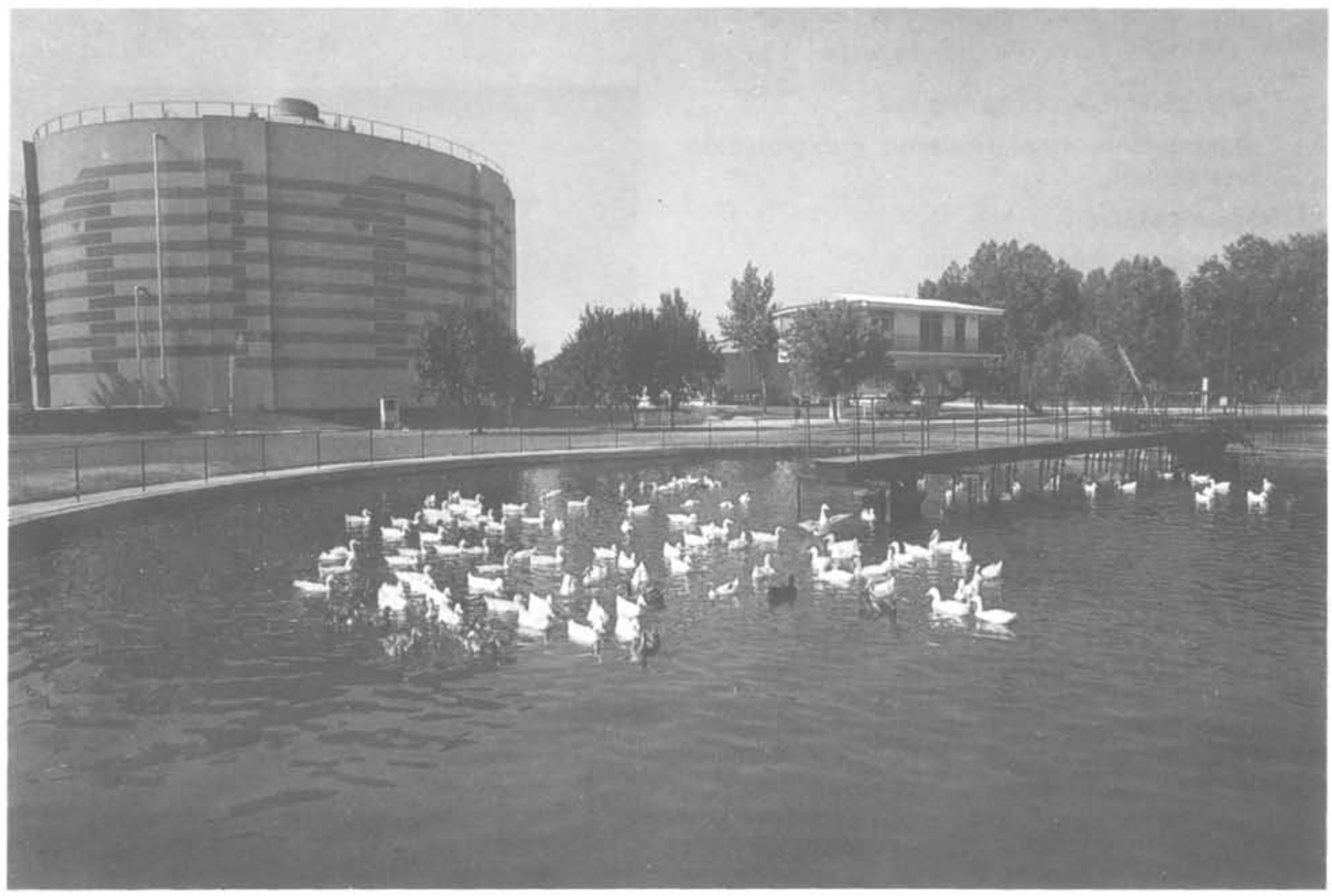

Balsa de decantación de la depuradora de Viveros. El pato ha sido identificado con el Plan de Saneamiento Integral de Madrid. 


\section{INTRODUCCION}

La fecha del 22 de septiembre de 1984, en que el rio Manzanares se pobló de peces y patos, marcó un momento de enorme significación en la historia de Madrid.

Un rio, que antes era una autèntica cloaca descubierta, se habia transformado en un cauce de aguas limpias en el que era posible la vida. Aquel hecho era la consecuencia más visible, a ojos de los madrileños, del Plan de Saneamiento Integral de Madrid.

Pero detrás de este acto simbólico se encerraba obviamente la culminación de una serie de acciones emprendidas durante varios años para conseguir que la ciudad completase su sistema de saneamiento.

Con este conjunto de acciones comprendidas en el Plan, puede decirse que esta infraestructura básica, siempre deficitaria en Madrid, ha adquirido ya el nivel de suficiencia capaz de prestar un servicio acorde con las auténticas necesidades de la Villa.

\section{LAS ACTUACIONES DEL PLAN}

A continuación expresaremos, a manera de prólogo, las lineas maestras en las que se ha desenvuelto el Plan, apoyándonos en lo que hemos venido definiendo siempre como Eotructura Técnica del mismo, es decir, clasificando las acciones en cinco apartados:

1. Construcción de obras nuevas.

2. Conservación, mantenimiento y explotación del sistema.

3. Reglamentación.

4. Acciones complementarias.

5. Investigación.

\section{Construcción de obras nuevas}

Ha sido éste el apartado más espectacular, hasta el punto de que en muchos casos, se ha identificado erróneamente el Plan con las obras realizadas, sin vislumbrar que éstas eran simplemente una parte del mismo; un medio muy importante, pero sólo un medio, como hemos indicado en varias ocasiones, para conseguir los fines globales del sistema.

Dentro de este apartado de obras nuevas distinguiremos las realizaciones llevadas a cabo en materia de alcantarillado y en depuración.

En ALCANTARILLADO, el objetivo general era el de completar la red en su esquema básico de colectores primarios y mejorar aquellos sectores de la red secundaria que presentaban deficiencias crónicas.

Con esta idea, los colectores de la red primaria construidos, han supuesto, por un lado, la canalización de vertidos de aguas residuales que discurrian a través de arroyos descubiertos, como son los casos de los colectores de Valdebebas, Rejas o Vicálvaro, y, por otro lado, la mejora de la capacidad de desagüe de algunas zonas de la ciudad, como los colectores del Fresno, Interceptor de la Margen Derecha, Axiles, etc.

En varios casos, estos colectores constituyen prolongación o doblado de otros ya existentes con anterioridad, que habian saturado su capacidad con el desarrollo de la ciudad.

Las secciones de todos ellos son visitables, con cuna y andén, y sus dimensiones varian según las capacidades hidráulicas necesarias, siendo de destacar en este sentido los nuevos colectores de Rejas, Sur y prolongación de la Margen Derecha del Manzanares, cuyas anchuras interiores alcanzan los $3 \mathrm{~m}$, con alturas de hasta 3,80 m sobre cuna en el caso del Colector Sur.

Las longitudes de estos colectores construidos en la red primaria también han sido variables y superan en su conjunto los 70 kilómetros, destacando por su desarrollo el colector de Valdebebas de más de $7 \mathrm{~km}$, y el nuevo de Rejas que supera los $9 \mathrm{~km}$.



Uno de los 150 colectores del Plan de Saneamiento Integral de Madrid. 


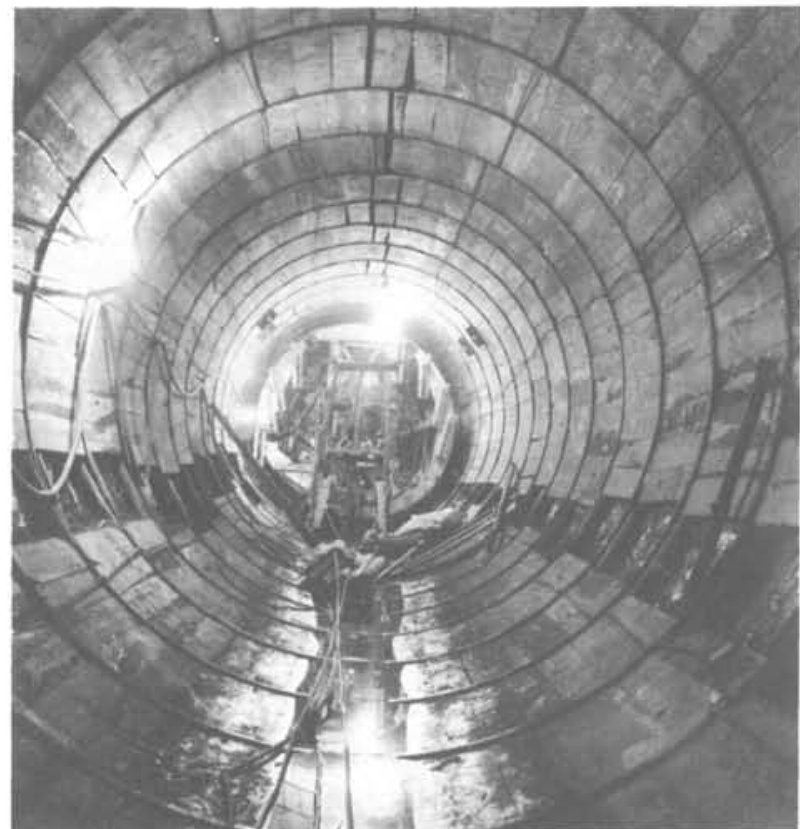

Construcción de un colector interruptor.

Los colectores construidos correspondientes a esta red primaria han sido los siguientes:

- Colector doblado del Axil del P.o de la Castellana y c/. Barquillo.

- Colector interceptor margen izquierda del Manzanares (tramo superior).

- Colector Sur (3.a fase).

- Colector Vicálvaro Superior.

- Colector doblado de Rejas.

- Colector de Barajas y adyacentes.

- Colector de Valdebebas (3 tramos).

- Colector Fresno Superior.

- Colector Avda. Victoria y Transvase a Viveros.

- Colectores Meaques, Galicia y Aluche Nuevo.

- Colector Interceptor Margen derecha del Manzanares.

- Colector Pradolongo, Perales, Abrantes.

Además de estas actuaciones en la red básica, se han llevado a cabo más de 150 obras en la red secundaria de alcantarillado con el fin de resolver los problemas más acuciantes de éste, actuando en aquellos puntos en los que sistemáticamente se venian produciendo inundaciones en época de tormenta o en aquellos otros en los que existian disfunciones notables en la red existente.

Han sido actuaciones puntuales en el alcantarillado de calles aisladas o en barrios completos que adolecian de defectos en su red o insuficiencias globales, e incluso que aún carecian de ella.
Con todos ellos se han superado los $200 \mathrm{~km}$ de actuación, que en su mayor parte se han concentrado en zonas periféricas.

Las secciones de las conducciones construidas en la red secundaria son en varios casos visitables o semivisitables, si bien en la mayor parte, y por su propia naturaleza local, se trata de alcantarillas tubulares de dimensiones variables entre $0,40 \mathrm{~m}$ y $1,00 \mathrm{~m}$.

La variedad de trazados de todos estos colectores y alcantarillas, construidas en el Plan y su dispersión por el término municipal han supuesto distintos condicionantes geotécnicos encontrándose todos los estratos de los suelos clásicos de la ciudad, desde rellenos hasta margas yesiferas, pasando por arenas, gravas, toscos y peñuelas.

Esta variabilidad de terrenos, junto a la impuesta por las condiciones hidráulicas, ha supuesto la utilización de diversas técnicas constructivas, tanto a cielo abierto como en mina.

Entre las actuaciones en la red secundaria, debemos destacar las siguientes:

- Colector Butarque (tramo intermedio).

- Colector Valcieza.

- Colector Fresno inferior.

- Colector Doblado de Pinos.

- Saneamiento Colonia Sta. Margarita.

- Colector margen derecha (2.0 tramo).

- Colector Barrio Salvador.

- Colector Eugenia Montijo - Gral. Franco.

- Colector General Aranaz.

- Colector Arroyo del Olivar.

- Saneamiento Colonia de Malmea

hasta un total de 150 actuaciones.

La actuación llevada a cabo en materia de DEPURACION se basó en establecer un sistema de plantas capaz de tratar el $100 \%$ de los caudales de aguas residuales de la ciudad con un nivel de depuracion tal que las aguas vertidas a los rios

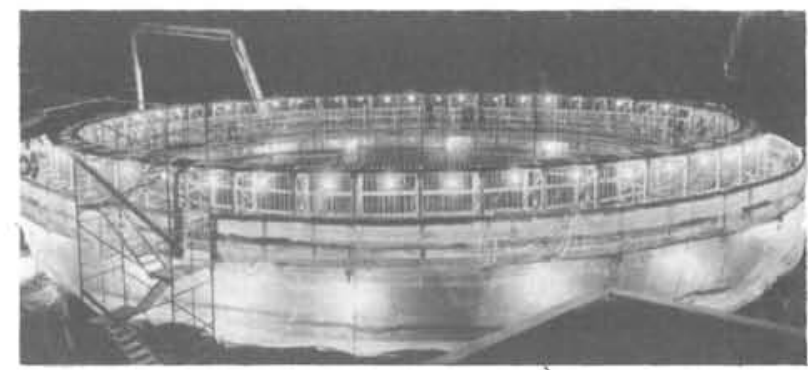

Digestor secundario. 




Complejo de estaciones depuradoras de Viveros. Esquema de funcionamiento.

no superasen la cifra de $20 \mathrm{ppm}$ tanto en $\mathrm{DBO}_{5}$ como en SS.

Las condiciones existentes en el comienzo del Plan influyeron obviamente en el esquema del sistema, dado que funcionaban instalaciones con tratamiento primario en tres casos (China, Rejas y Butarque) y se habian iniciado obras también de 1.a fase en otro caso (Sur).

Asimismo, la antigua planta de viveros funcionaba con insuficiencia de capacidad y con rendimientos inferiores al nivel propuesto, pero disponian de terrenos adyacentes de dominio municipal.

Estas cinco subcuencas determinaron un planteamiento consistente en ampliar o renovar las instalaciones existentes e implantar dos nuevas estaciones en las subcuencas restantes.

De esta forma surge el nuevo sistema con base a lo siguiente:

- Ampliar a tratamiento biológico las EDAR de China, Rejas y Butarque.

- Reformar y adecuar las instalaciones existentes (primario) de estas plantas.

- Construir una nueva planta en viveros, dejando la antigua para tratamiento de afino.

- Ampliar a tratamiento biológico la EDAR Sur, que entonces se encontraba en la 1. a fase de construcción.

- Construir las nuevas plantas de Valdebebas y Suroriental.

La fijación de los dimensionamientos de las plantas se estableció en base a una dotación de abastecimiento de $385 \mathrm{l} / \mathrm{hab} /$ dia y a un horizonte de población coincidente con el límite de previsiones del Plan de Ordenación. Sin embargo, para la etapa del Plan de Saneamiento Integral se propusieron unas capacidades de tratamiento suficientes para atender las necesidades durante un periodo de tiempo suficientemente amplio, sin alcanzar la situación límite.
En base a ello y teniendo en cuenta criterios de modulación de las posibles ampliaciones en el futuro se dispusieron las capacidades con las que se ha construido el sistema, que se indican a continuación:

Manzanares:

Viveros ................ $2,1 \mathrm{~m}^{3} / \mathrm{seg}$

La China . . . . . . . . . . . . . . $3,3 \mathrm{~m}^{3} / \mathrm{seg}$.

Butarque................ $3,2 \mathrm{~m}^{3} / \mathrm{seg}$.

Sur ............. (primario) $6,0 \mathrm{~m}^{3} / \mathrm{seg}$.

Sur ............ (secundario) $4,0 \mathrm{~m}^{3} / \mathrm{seg}$.

Suroriental .............. $0,4 \mathrm{~m}^{3} / \mathrm{seg}$.

Jarama:

Valdebebas .............. $0,4 \mathrm{~m}^{3} / \mathrm{seg}$.

Rejas ................ 1,2 $\mathrm{m}^{3} / \mathrm{seg}$

\section{Conservación, mantenimiento y explotación del sistema}

La infraestructura construida en el Plan y descrita en el apartado anterior es la base para proporcionar un servicio que debe estar apoyado en una estructuración técnica y económica que asegure permanentemente estas prestaciones.

En el aspecto técnico, con el Plan se estructura un Servicio Técnico Municipal que especificamente se ocupa de estas competencias: el Departamento de Agua y Saneamiento, encuadrado en los Servicios de Medio Ambiente del Area de Urbanismo e Infraestructuras.

Los modelos de gestión aplicados a la conservación y explotación del sistema son diferentes en el caso del alcantarillado y la depuración.

En el caso de la depuración, el sistema puesto en práctica tiene la fórmula de empresas mixtas, de forma que para cada una de las Estaciones Depuradoras se ha constituido una de ellas, con participación municipal del $30 \%$ y privada en el $70 \%$.

La concesión del mantenimiento, conservación y explotación se ha hecho a cada una de estas empresas mixtas por un periodo de 5 años, concursándose la participación que corresponde a la parte privada.

En el caso del alcantarillado, la conservación y reparación se gestiona por el sistema de empresas privadas, también por un período de 5 años.

La ciudad se ha dividido, a este efecto, en tres zonas, denominadas $\mathrm{A}, \mathrm{B}$ y $\mathrm{C}$, que coinciden con las subcuencas correspondientes al sistema en la forma siguiente:

ZONA A: Subcuenca de Valdebebas, Rejas y Viveros (M. Izquierda). 
ZONA B: Subcuenca de Butarque y Viveros (M. Derecha).

\section{ZONA C: Subcuenca de China, Sur y Suroriental.}

En cada zona, la empresa correspondiente, adjudicatoria del servicio por concurso, asume los trabajos de limpieza de la red y conservación, tanto preventiva como curativa.

En todos los casos, el Servicio Técnico Municipal, a través de las Secciones correspondientes del Departamento de Agua y Saneamiento, asume la inspección y el seguimiento de los trabajos que desarrollan tanto las Empresas Mixtas como las privadas encargadas de cada servicio.

Otro aspecto abordado por el Plan de cara a la explotación, es el referido al destino final de los fangos producidos en las distintas depuradoras.

En cinco de estas depuradoras, el destino agricola de los fangos es asumido por dos Empresas privadas que se encargan de su retirada, manipulación y comercialización. En las dos depuradoras restantes, Butarque y Suroriental, en la que por su naturaleza se consideró la conveniencia de depositarlos en otro vertedero, es otra Empresa privada la que se encarga del transporte y depósito.

En estos casos, también, las concesiones a cinco años, se adjudicaron mediante concurso y se controlan por el Departamento Municipal.

Hay, además, otros aspectos de cara a la explotación del sistema que el Plan ha desarrollado. Algunos de ellos, simultáneamente, con la ejecución de las obras realizadas, o, por mejor decirlo, anteriormente, al estar incluidos en los propios diseños o en los Pliegos de Condiciones de las instalaciones.

Nos referimos, por citar algunos casos concretos, a las instalaciones de control informático de las Depuradoras, o las instalaciones de recuperación de energia, que seis de las siete plantas poseen.

Son éstos y algunos otros, criterios que primaron en su momento, y que, por ello, forman hoy parte del sistema, que desde un principio se planteó con una perspectiva preferentemente hacia la explotación a costa, a veces, de una mayor inversión inicial.

No quisiéramos olvidar la preferente atención que el Plan y el Departamento dedica al rio Manzanares, cuya imagen es, en gran medida, un contraste ante el ciudadano de la gestión del saneamiento de Madrid, aunque su tramo urbano solamente depende del funcionamiento de una parte del sistema.
El mantenimiento del rio en las condiciones adecuadas, y en su aspecto visual, está encomendado, dada su intima dependencia, a la misma Empresa Mixta, encargada de la Depuración de Viveros.

\section{Reglamentación}

Es éste un tercer aspecto contenido en la estructura del Plan que también se ha abordado, entendiendo que constituye un complemento indispensable del mismo.

La publicación de una Ordenanza relacionada con los vertidos no domésticos al sistema, completan la tripleta de sus lineas básicas.

El texto de dicha Ordenanza se transcribe integramente en la sección de Normativa del presente número de esta revista.

\section{Acciones Complementarias}

Otro grupo de actuaciones, menos relacionadas con el saneamiento de las aguas residuales, pero si ligadas a otros aspectos de la vida de la ciudad, de su estética y desde luego relacionadas con el liquido elemento, han formado también parte del P.S.I.M.

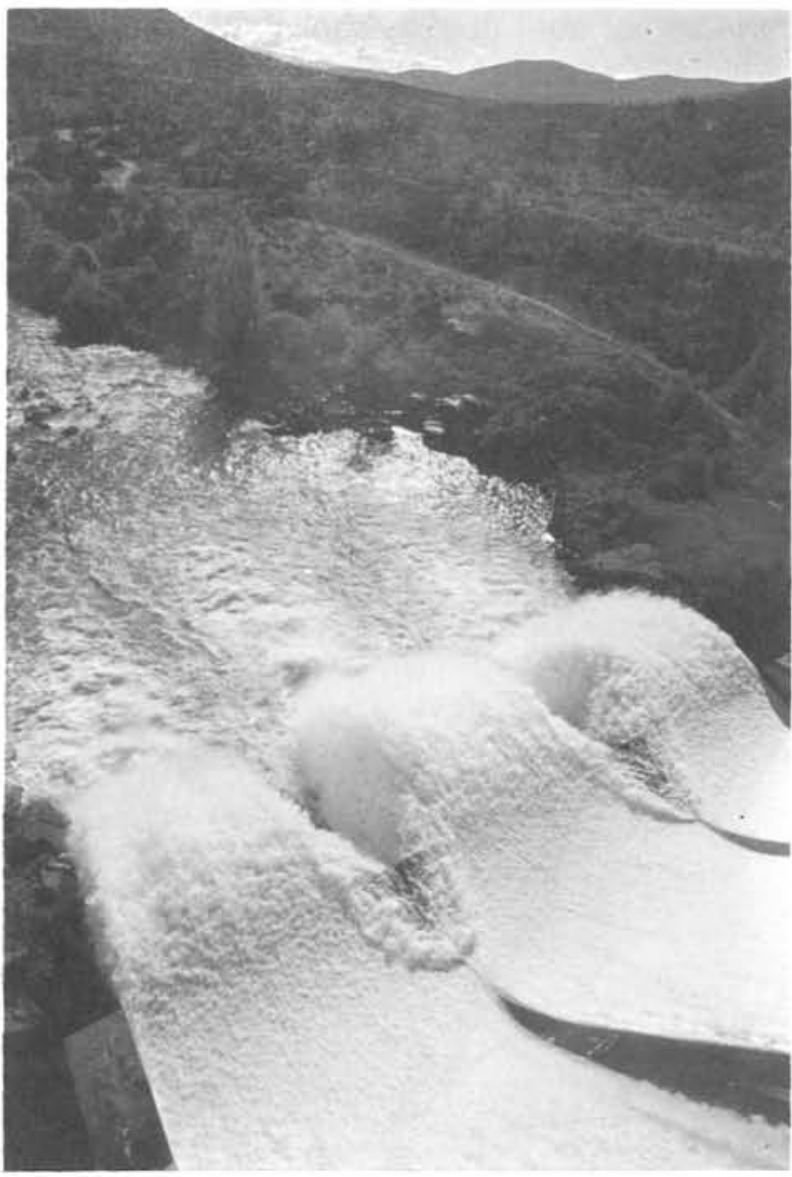

Aliviadero de una de las presas que rodea Madrid. 
Nos referimos a la actuación desarrollada en los lagos, estanques y fuentes ornamentales de $\mathrm{Ma}$ drid.

Asi, tanto el Lago de la Casa de Campo, como el Estanque del Parque del Retiro, han sido objeto de atención concreta realizando obras de adecuación y limpieza y dotándoles de sendas estaciones de tratamiento de sus aguas, de forma que hoy dia son utilizadas con una mayor garantía de salubridad.

En varias fuentes de la ciudad de carácter ornamental, se ha venido actuando, asimismo, adecuando sus instalaciones eléctricas e hidráulicas, que en muchos casos se encontraban en deficiente estado. Pero, quizá, el aspecto más novedoso lo constituya el establecimiento de un servicio de conservación de estos estanques y fuentes.

\section{Investigación}

Finalmente, queremos hacer mención de este quinto apartado que se planteó en su momento como interesante del Plan, y que implica una vocación por parte del Ayuntamiento de Madrid y, del propio Departamento de Agua y Saneamiento, en colaborar con aquellos Organismos, Entidades, Empresas o Investigadores que día a dia trabajan por abrir nuevos caminos o nuevos conocimientos en este campo.

En este tipo de colaboración, se han inscrito convenios de investigación con el Instituto $\mathrm{Na}$ cional de Investigaciones Agronómicas (INIA), para analizar el contenido de metales en fangos de depuradoras y su aplicación agricola, con la Escuela de Ingenieros Agrónomos de Madrid, para el análisis de los procesos de digestión, con la Environal Protection Ambiente (EPA) de U.S.A. y la Dirección General del Medio Ambiente del MOPU, para investigación de los procesos

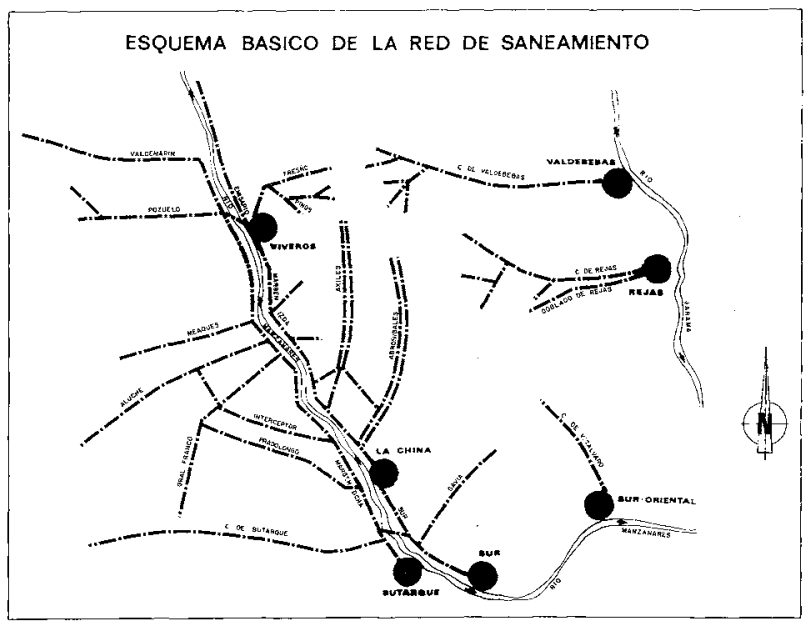

microbiológicos en fangos, con el Centro de investigaciones del Agua (CSIC) para mejora de tratamientos en plantas biológicas, etc., estando en proceso de estudio varias otras propuestas.

Además de ello, en nuestras instalaciones y laboratorios se han tutelado numerosas tesis doctorales o trabajos concretos de titulados y alumnos de distintas especialidades profesionales.

\section{LA INFRAESTRUCTURA ACTUAL DEL SISTEMA}

Con las obras ejecutadas en el PSIM, la infraestructura de Saneamiento de la ciudad responde al esquema siguiente:

El alcantarillado es de tipo unitario y gravitatorio, con un desarrollo lineal superior a los $3.200 \mathrm{~km}$ de dominio municipal. De éstos, el $40 \%$ aproximadamente es visitable, en tanto que el $60 \%$ restante es tubular o no visitable.

Las secciones tubulares varian entre diámetros de 0,30 m y 1,00 m, correspondiéndose el mayor porcentaje (aproximadamente el $75 \%$ ) con los diámetros menores $(\varnothing 30$ y $\varnothing 40)$.

Las secciones visitables son variables, desde las semivisitables o gateras que apenas suponen el $7 \%$ hasta los grandes colectores de alturas libres entre 3 y 4 metros, que también suponen un porcentaje pequeño, algo superior al $2 \%$. Las secciones visitables más usuales son las de alturas libres comprendidas entre $1,60 \mathrm{~m}$ y $1,85 \mathrm{~m}$, que representan aproximadamente el $70 \%$ total.

El vertido del alcantarillado se efectúa a dos cuencas: la del Manzanares y la del Jarama en una proporción aproximada del $90 \%$ y el $10 \%$ en tiempo seco.

El desagüe a los rios citados en tiempo seco se verifica en siete puntos, cinco de ellos en el río Manzanares y dos en el río Jarama, correspondiéndose estos siete puntos con la situación de las siete estaciones depuradoras de la ciudad, donde todas las aguas residuales son sometidas a un tratamiento primario y biológico antes de su entrega al cauce receptor.

Para la descarga de la red en tiempo de aguacero, el sistema dispone de aliviaderos en los coiectores importantes siendo dignos de mencionar los existentes en los colectores marginales del Manzanares. 


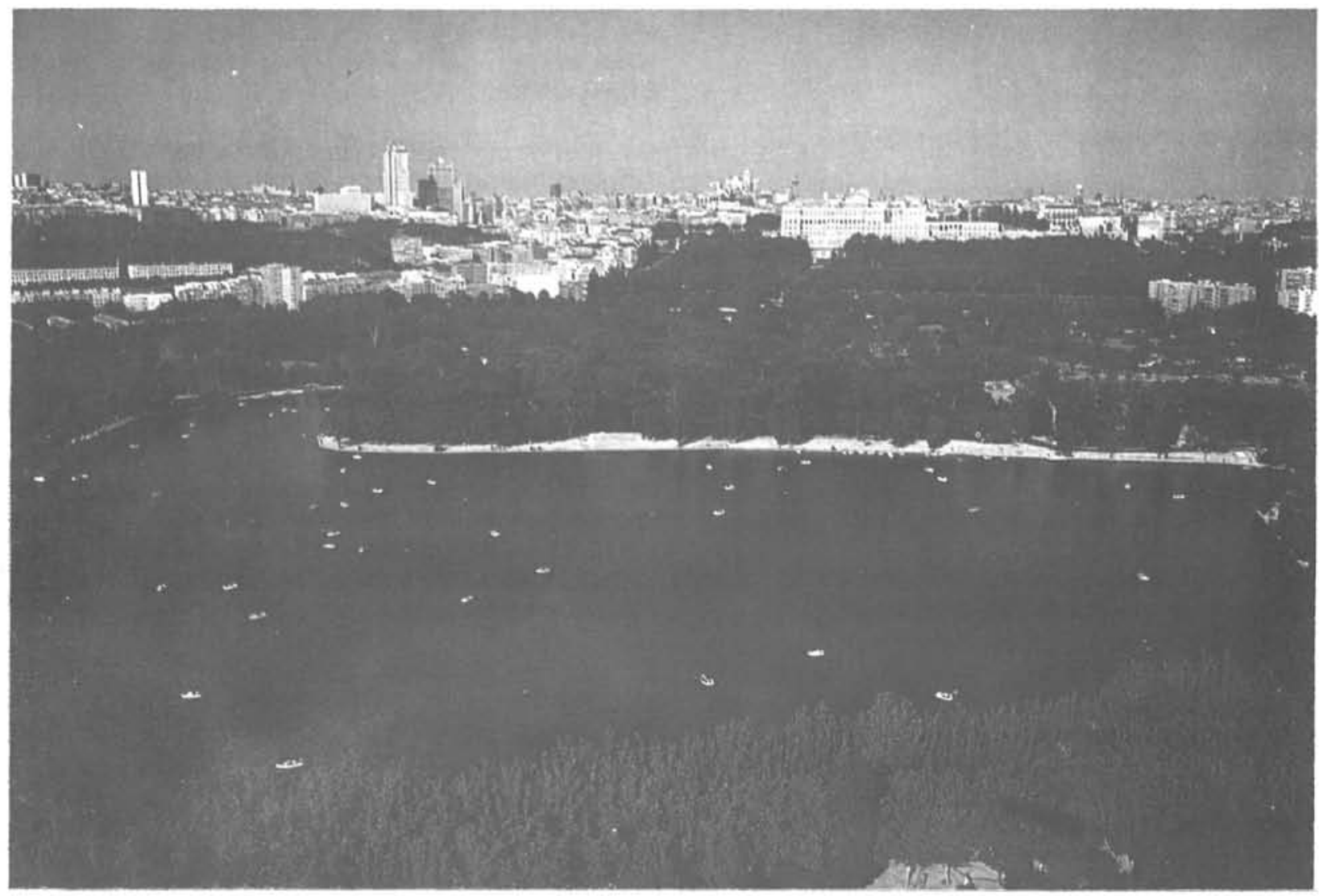

Lago de la Casa de Campo.

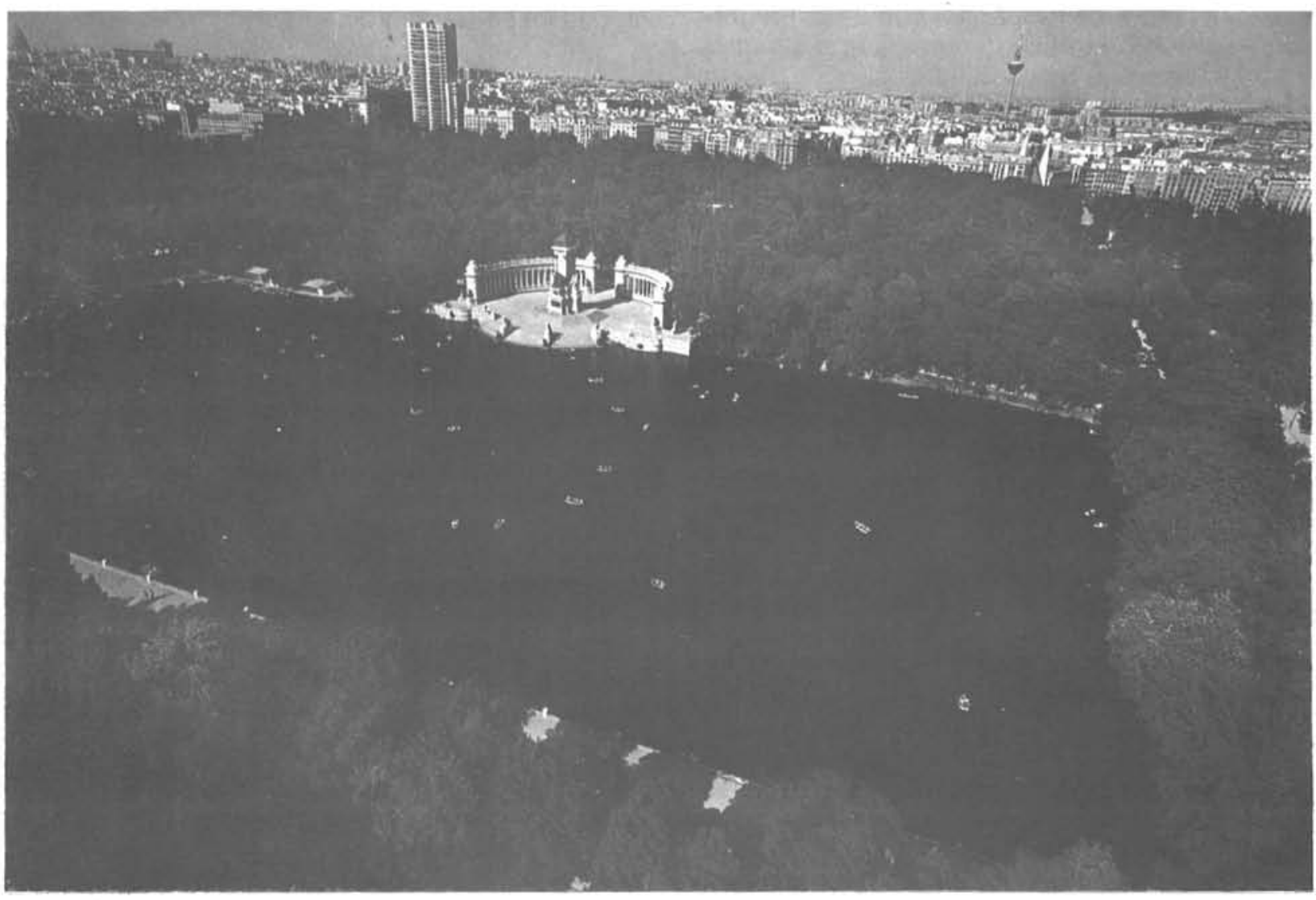

Estanque del Parque del Retiro. 
Las siete subcuencas en que queda dividido el sistema tienen las siguientes caracteristicas:

\section{Subcuenca de Viveros}

Está dividida en dos partes: una de ellas corresponde a la margen izquierda del río Manzanares, como desagüe natural y recoge los vertidos de El Pardo, Peña Grande, Tetuán, parte de Fuencarral, etc.

Su colector principal es el Colector del Fresno que desemboca en la E.D.A.R. de Viveros.

La otra parte de esta subcuenca puede decirse que es artificial dado que al estar situada en la margen derecha, su desagüe natural seria hacia este lado.

La existencia de La Casa de Campo, determinó en el PSIM, la construcción de un transvase cruzando superiormente el rio Manzanares, para así tratar estas aguas en la Planta de Viveros y evitar la construcción de un colector, a través de este Parque, solución que habria supuesto una inversión muy elevada y que lo había deteriorado en gran medida.

La Depuradora de Viveros es una instalación critica del sistema dada su ubicación en el comienzo del tramo urbano del Manzanares, cuyas aguas proceden básicamente de esta Depuradora. La existencia de fauna, así como las exigencias de carácter estético, obligan a una atención permanente de sus rendimientos.

Es la única planta dotada de un tratamiento complementario de afino para reducción de nitrógeno amoniacal.

\section{Subcuenca de La China}

Comprende todo el centro de la ciudad y su colector principal es la Margen Izquierda del Manzanares. Los colectores principales de esta subcuenca son los Axiles de la Castellana, Abroñigales de la $\mathrm{M}-30$, así como la serie de perpendiculares al río que constituyen un esquema en forma de peine.

Las aguas residuales de esta subcuenca, hasta un caudal de $3,3 \mathrm{~m}^{3} / \mathrm{seg}$, se tratan en la EDAR China, traspasándose el exceso sobre este caudal a la Depuradora Sur.

\section{Subcuenca Sur}

Esta subcuenca comprende los colectores de la zona sudeste de Madrid, abarcando parte de Vallecas y la denominada zona de La Gavia.
La Depuradora Sur, correspondiente a ella trata estos caudales y los procedentes del centro de Madrid, que superan el limite establecido en la EDAR China.

Se puede, por tanto, considerar esta subcuenca como solidaria de la anteriormente referida.

\section{Subcuenca de Butarque}

Es la única subcuenca situada en la margen derecha del Manzanares. Sus colectores principales son la Margen Derecha y el nuevo Interceptor.

En espina, reciben estos colectores las aportaciones de una serie de colectores situados al sur de la Casa de Campo, como son Meaques, Aluche, Valdecelade, General Ricardos, Pradolongo, Villaverde y Butarque.

Sus aguas se tratan en la EDAR de Butarque, existiendo un transvase sobre el Manzanares que puede enviar caudales a la margen izquierda para su posible tratamiento en la EDAR Sur.

\section{Subcuenca Suroriental}

Corresponde a las zonas de Vicálvaro y adyacentes y sus aguas se tratan en la EDAR Suroriental, a la que también llegan algunas aportaciones de Rivas de Vaciamadrid.

\section{Subcuenca de Valdebebas}

El eje fundamental de esta subcuenca es el Colector de Valdebebas que recibe aportaciones de varios núcleos urbanos e industriales adyacentes.

Este colector discurre en sentido oeste-este por todo el norte del término municipal recogiendo las aguas de Manoteras, Virgen del Cortijo, Barajas, etc.

Sus aguas se tratan en la EDAR de Valdebebas con vertido al Jarama.

\section{Subcuenca de Rejas}

Recibe los vertidos de la zona este de la ciudad. Los colectores principales son los dos de Rejas, antiguo y nuevo,y las aportaciones a ellos proceden de los barrios de Ciudad Lineal, Hortaleza, Canillas, Canillejas, San Blas, etc. que, a través de los colectores denominados Santo, Quinto, Boltania, etc., los conducen al principal.

Sus aguas se tratan en la EDAR de Rejas que vierte al Jarama. 


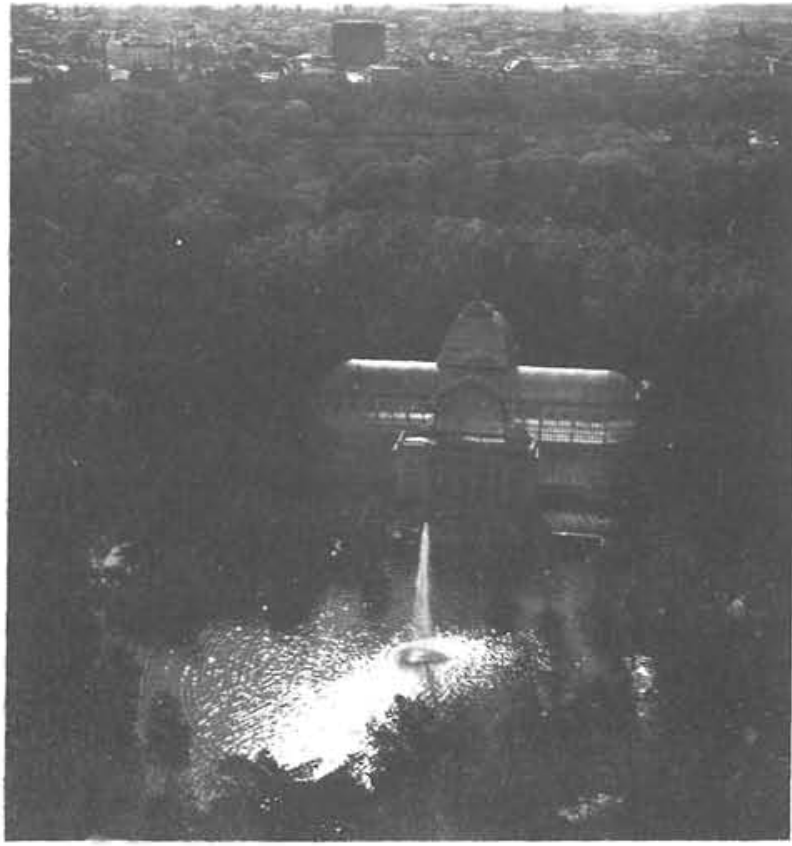

Palacio de Cristal, en el Parque del Retiro.

\section{Conexiones}

Como se ha descrito, el sistema está conectado entre si en cuanto a las Depuradoras que vierten al rio Manzanares, con excepción de la Suroriental.

Asi, las aguas de la Subcuenca de Viveros pueden, en caso conveniente, conducirse hasta la EDAR China e incluso a la EDAR Sur sin tratarse en Viveros o, incluso, después de su tratamiento, sin efectuar el vertido en dicho punto.

Igualmente sucede con las aguas de la Subcuenca de Butarque que pueden conducirse a la EDAR Sur, o las de La China que pueden seguir idéntico camino.

El papel de depuradora de cola adjudicado a la EDAR Sur, y la conexion entre las plantas más importantes citadas, confiere al sistema una gran flexibilidad para momentos de emergencia o para situaciones de limpieza o reparación en alguna de estas plantas.

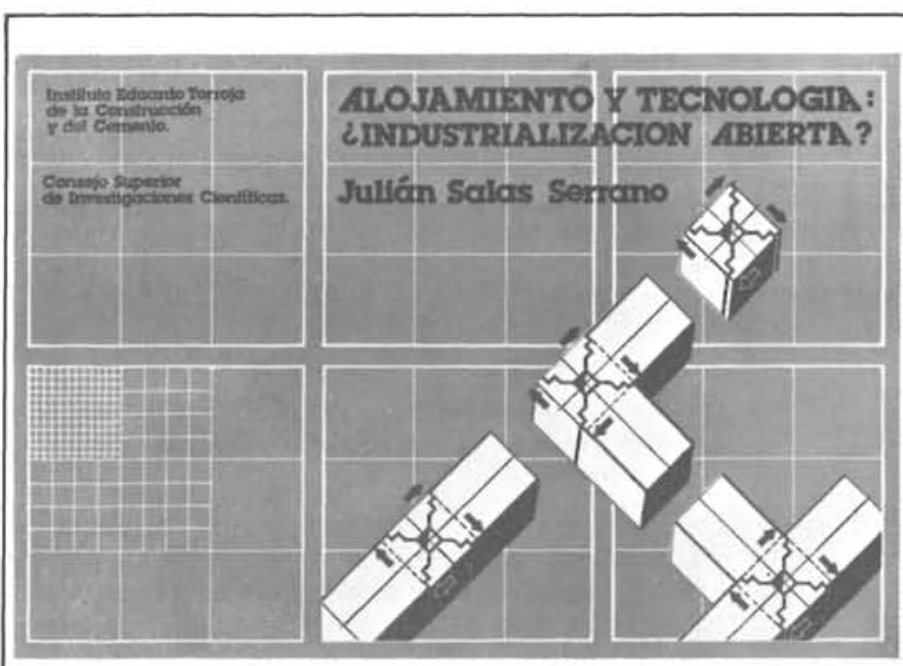

\section{ALOJAMIENTO Y TECNOLOGIA: ¿INDUSTRIALIZACION ABIERTA?}

JULIAN SALAS, ING. IND. (I.E.T.c.c.)

Un volumen de 160 páginas, 109 figuras y 16 tablas. Tamaño $240 \times 168 \mathrm{~mm}$. Encuadernado en rústica. Precios: España, 1.200 ptas; extranjero, 17 \$ USA.

\section{SUMARIO:}

Prólogo Prof. G. Ciribini.

\section{Introducción}

Capítulo 1.- La industrialización en las proclamas y manifiestos de arquitectura.

Capítulo 2.-¿Réquiem por la construcción industrializada?

Capítulo 3.-Algunos conceptos básicos.

Capítulo 4.-¿Proyecto tradicional, construcción industrializada?

Capítulo 5.-Componentes.

Capítulo 6. - La coordinación dimensional hoy.

Capitulo 7.-Flexibilidad, intercambiabilidad y catálogos.

Capítulo 8.--Industrialización, normativa y calidad.

Capitulo 9.-Reflexiones finales.

publicación del

INSTITUTO EDUARDO TORROJA 


II Concurso
lberoamericano
de Informes
1986

Promovido por la Revista INFORMES DE LA CONSTRUCCION y patrocinado por la Asociación Española de Constructores internacionales. el Consejo Superior de investigaciones Científicas, el instituto de Cooperación Iberoamericana y la Junta de Construcciones. instalaciones y Equino Escolar del Ministerio de Educación y Ciencia.
Información y recepción: Instituto "EDUARDO TORROJA" Apartado 19.002. Tfno: 2020440 28080 Madrid. España

\section{PRESENTACION:}

La revista INFORMES DE LA CONSTRUCCION, con 38 años de existencia y amplia difusión en los páises iberoamericanos, desea sumarse a la conmemoración del $V$ Centenario del Descubrimiento dedicando, con carácter bienal, sucesivos números extraordinarios a temas específicos del ámbito de la construcción en Iberoamerica. A tal fin se convocan sucesivos Concursos, hasta un total de cinco, el último de los cuales coincidirá, en 1992, con.la celebración del V Centenario.

El fin primordial de estos Concursos es el de estrechar los vínculos entre los profesionales, empresas e instituciones de habla castellana del ámbito de la construcción y afines, facilitando, mediante la Revista INFORMES, el intercambio de conocimientos y puntos de vista respecto a aspectos fundamentales del hecho constructivo.

El Primer Concurso beroamericano de informes sobre el tema "SOLUCIONES ACTUALES AL PROBLEMA DE LA VIVIENDA EN IBEROAMERICA» se falló el 20 de noviembre de 1984.

\section{Bases:}

1 OBJETO: Se pretende seleccionar un conjunto de trabajos inéditos oue habrán de constituir un número monográfico dedicado a lberoamérica.

2 TEMA: El temia de este número monográfico será: «SOLUC10NES ACTUALES AL PROBLENIA DE LAS CONSTRUCCIONES DOCENTES EN IBEROAMERICA».

3 CONTENIDO: Quedarán en libertad los autores en la elección del enfoque que deseen dar a sus artículos: trabajos de investigación, de planificación, exposición de proyectos, obras realizadas, tecnologías de materiaies autóctonos, etcétera. La única limitación consiste en ceñirse al tema propuesto.

4 PRESENTACION: La forma de presentación deberá seguir las normas habituales de la revista, las cuales se reproducen en el reverso. Los trabajos serán redactados en casteflano y no excederán de 30 páginas, mecanografiadas a doble espacio, en hojas UNE A4.

5 PARTICIPANTES: Podrán tomar parte en el concurso tanto personas físicas como jurídicas (cátedras, grupos de trabajo, etc.) pertenecientes a cualquier país del área iberoamericana, con excepción de España.

6 JURADO: Estará compuesto por las siguientes personas:

- Eduardo Aranguren Gárate, director general de la Asociación Española de Constructores Internacionales.

- Don José Calavera Ruiz, catedrático de la E.T.S. de Ingenieros de Caminos, Canales y Puertos de Madrid.

- Don Francisco Garcia de Paredes, jefe del Gabinete Técnico de la Junta de Construcciones del Ministerio de Educación y Ciencia.

- Don Eduardo Lechuga Jiménez, director de Cooperación Científico-Técnica del Instituto de Cooperación Iberoamericana.

- Don Salvador Pérez Arroyo, catedrático de la E.T.S. de Arquitectura de Madrid.
- Don Leandro Quintana Uranga, arquitecto venezolano ganador del Primer Concurso.

Por parte del Instituto Eduardo Torroja:

- Don Alvaro García Meseguer, director de INFORMES.

- Don Julián Salas Serrano, coordinador del Segundo Concurso.

7 PLAZO: El plazo de recepción de trabajos expira el 30 de agosto de 1986, admitiéndose los que lleguen a poder de la revisła antes del 30 de septiembre, con matasellos del pais de procedencia anterior al 30 de agosto.

8 FALLO: El tallo del jurado se hará público antes de finalizar 1986 y se comunicará a todos los autores de trabajos admitidos.

9 PREMIOS: Se establecen cinco premios, dos de los cuales podrán ser excepcionalmente declarados desiertos. La cuantía será la siguiente:

- Primer premio de 300.000 ptas. y pasaje para que el autor, o uno de los autores, viaje al acto de entrega de premios desde la capital del país de procedencia a Madrid, con estancia de una semana, por cuenta del Concurso.

- Segundo premio de 150.000 ptas.

- Tercer, cuarto y quinto premios de 75.000 ptas. cada uno. Los cinco trabajos premiados se publicarán en el número extraordinario de la revista, recibiendo sus autores diez ejempiares de la misma.

Los autores de todos los trabajos admitidos al concurso tendrán derecho a una suscripción anual gratuita de la revista INFORMES, reservándose ésta el derecho de publicar cualquier trabajo no premiado, en las condiciones habituales establecidas por la revista.

10 Clausula finAL: El material recibido no será devuelto. La presentación de un trabajo significa la plena aceptación de las bases de este Concurso.

\section{COORDINACION:}

